\title{
Coupling a Nanomechanical Resonator to a Cooper-Pair-Box Qubit
}

\author{
Matt LaHaye $^{1}$, Junho Suh ${ }^{1}$, Pierre Echternach ${ }^{2}$, Keith Schwab $^{3}$, $\underline{\text { Michael Roukes }}^{1}$ \\ ${ }^{1}$ Kavli Nanoscience Institute, Caltech, Pasadena, CA 91125 USA \\ ${ }^{2}$ Center for Space Microelectronics Technology, JPL, Pasadena, CA 91109 USA \\ ${ }^{3}$ Department of Physics, Cornell University, Ithaca, NY 14852 USA
}

\begin{abstract}
We demonstrate dispersive coupling between a Cooper-pair box (CPB) qubit and a VHF NEMS (nanoelectromechanical systems) resonator. The observed interaction strength is sufficient to pursue more advanced experiments to elicit quantum behavior in NEMS.
\end{abstract}

Recent progress in UHF NEMS [1], along with the demonstration of SET-based NEMS transduction [2,3], has opened up a way to explore quantum mechanics in macroscopic mechanical degrees of freedom. This invokes the question of how actually to measure the quantum nature of the NEMS. One possible set of measurement tools include superconducting electronics like the Cooper-Pair-Box (CPB) qubit, flux/phase qubits, or Superconducting SET(SSET) whose properties are dominated (in the case of the CPB) or in large part determined (in the case of the SSET) by quantum mechanics. In fact, SSET's have been operated near the quantum limit for continuous, linear displacement detection, even allowing for the observation of the influence of the SSET's quantum noise on the behavior of the NEMS [4]. Displacement detection with the SSET could ultimately lead to the production of ultracold NEMS modes [5], or even "squeezed" NEMS states [6]. However the observation of a nanoresonator existing in a superposition of position states or undergoing a quantum jump might require coupling to quantum mechanically "coherent" systems like the CPB. Our results measuring the dispersive interaction between CPB and NEMS are the first step toward these objectives.

The interaction between the NEMS and CPB qubit is achieved via electrostatic coupling (Fig. 1). The capacitance between the CPB island and a nearby, metallized nanoresonator is modulated through the displacement of the resonator about its equilibrium position. When a DC voltage bias is applied to the resonator, modulation of the capacitance results in modulation of the potential of the CPB island. For typical parameters [7], the interaction is characterized as dispersive, meaning that one should observe a change in the NEMS frequency, $\Delta \mathrm{f}_{\mathrm{NEMS}}$, that is determined by the CPB state.

We use a capacitive detection scheme here in order to measure the frequency shift [8]. The NEMS is biased at a DC potential Vg with respect to a nearby gate. The motion of NEMS modulates the capacitance between the gate and NEMS, in turn changing the charge on the gate. By calculating the current-voltage relation of this DC biased NEMS, we get a series RLC circuit as the equivalent circuit model. For typical dimensions, its resistance is on the order of mega-ohms, which we transform down to 50 Ohms using a resonant matching circuit (LC network). Fig. 2 shows the detection scheme (left) and an example of measured reflected voltage signal (right) around the mechanical resonance.

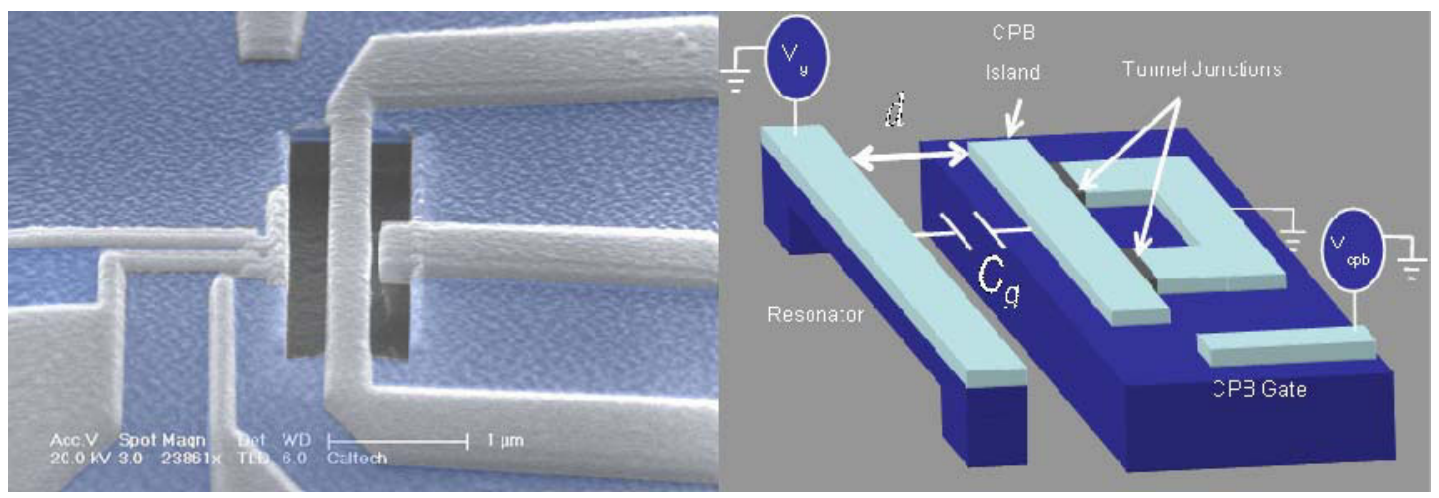

Fig. 1 SEM picture of the device(left) and electrostatic coupling scheme(right) 


\section{JMA3.pdf}
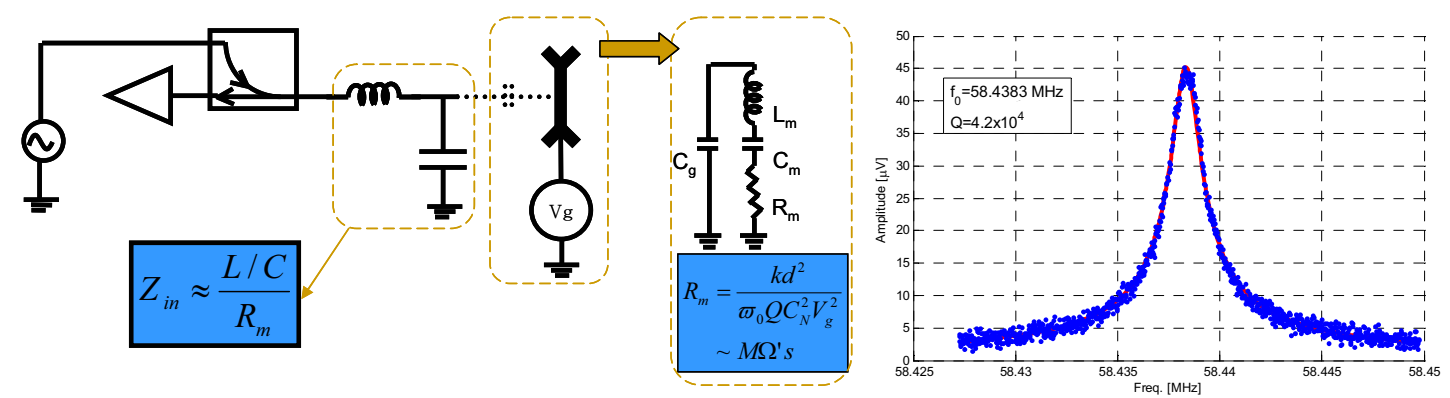

Fig. 2 (left) Capacitive detection circuit. The parameters in the inset are, $\mathrm{k}=($ effective spring constant), $\mathrm{d}=($ distance between gate and NEMS), $\omega_{0}=\left(\right.$ resonance frequency in $\mathrm{rad} / \mathrm{sec}$ ), $\mathrm{Q}=\left(\right.$ quality factor), $\mathrm{C}_{\mathrm{N}}=$ (gate-NEMS capacitance). (right) Mechanical resonance. The red curve is a Lorentzian fit.

We use slope-detection to measure $\triangle \mathrm{f}_{\text {NEMS }}$ while sweeping the CPB gate voltage and the flux through the CPB loop, (Fig. 3). It clearly shows the expected periodicity in gate charge and flux. By comparing with a simple model that incorporates i) electrostatic coupling between NEMS and CPB, ii) thermal activation of CPB when the Josephson energy is comparable to $\mathrm{k}_{\mathrm{B}} \mathrm{T}$ and iii) gate charge modulation by NEMS motion $\left(0.1 \mathrm{e}_{\mathrm{rms}}\right)$, we estimate the coupling strength to be $\sim 1.4 \mathrm{MHz}$. This is promising for proposed CPB-based ground-state cooling of NEMS [9], NEMS Fock states [7], and NEMS superposition states [10].
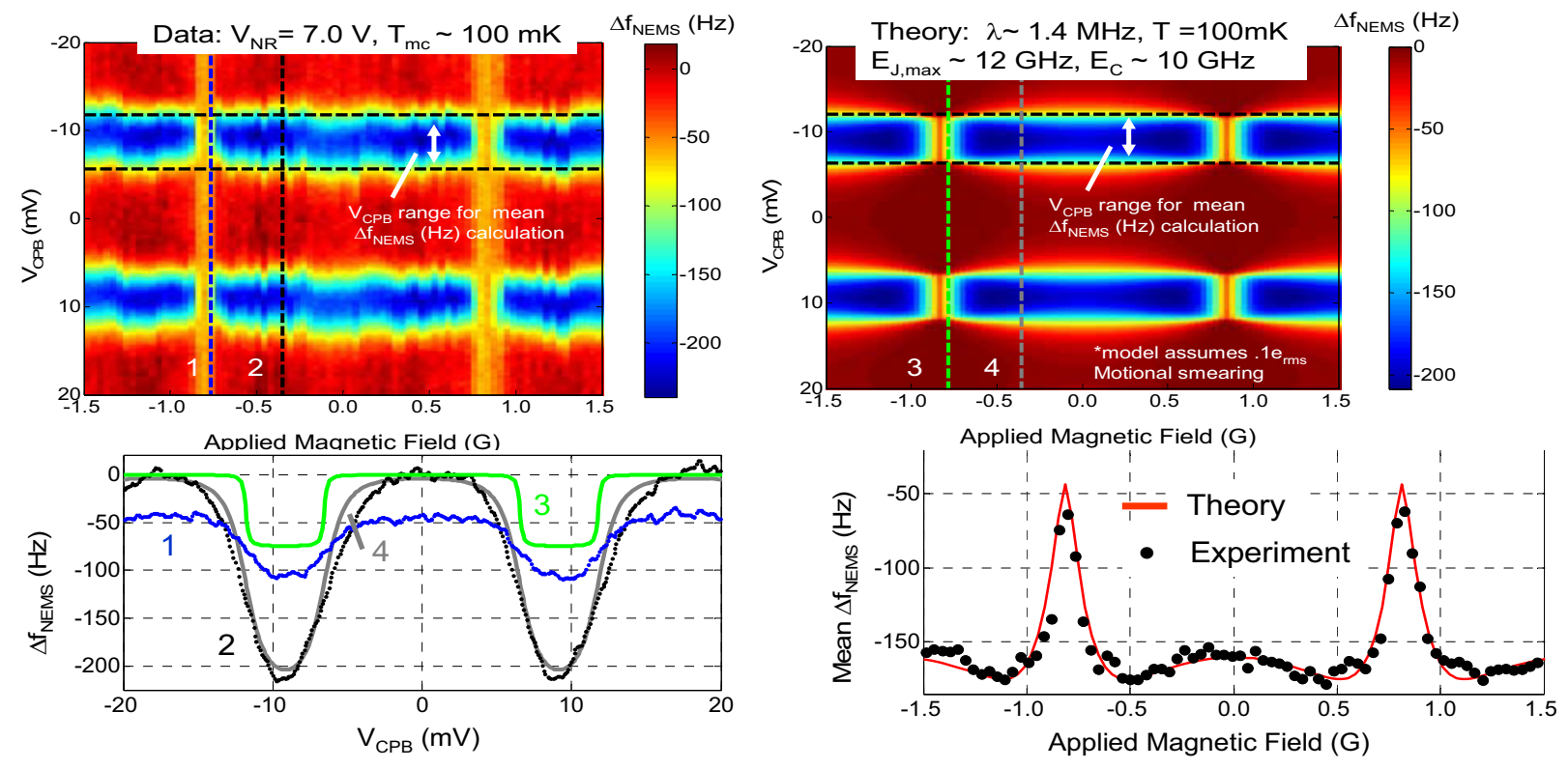

Fig. 3. Frequency shift, $\Delta \mathrm{f}_{\text {NEMS }}$, map in CPB gate voltage - magnetic field space

[1] X.M.H Huang et al., "Nanodevice motion at microwave frequencies,"Nature 421, 496 (2003).

[2] R.G. Knobel and A. N. Cleland, "Nanometer-scale displacement sensing using a single-electron transistor," Nature 424, 291-293 (2003).

[3] M.D. LaHaye, O. Buu, B. Camarota, and K.C. Schwab, “Approaching the quantum limit of a nanomechanical resonator," Science 304, 74-77 (2004).

[4] A. Naik et al., "Cooling a nanomechanical resonator with quantum back-action," Nature 443, 193-196 (2006).

[5] A. Hopkins, K. Jacobs, S. Habib, and K.C. Schwab, "Feedback cooling of a nanomechanical resonator," Phys. Rev. B 68, 235328/1-10 (2003).

[6] R. Ruskov, A.N. Korotkov, and K.C. Schwab, "Quantum nondemolition squeezing of a nanomechanical resonator," IEEE T. Nanotechnol.4, $132-140(2005)$.

[7] E.K. Irish and K. Schwab, "Quantum measurement of a coupled nanomechanical resonator-Cooper-pair box system," Phys. Rev. B 68, 155311/1-7 (2003).

[8] P.A. Truitt et al., "Efficient and sensitive capacitive read-out of nanomechanical arrays," Nano Letters 7, 120-126 (2007).

[9] I. Martin, A. Shnirman, L. Tian and P. Zoller, "Ground-state cooling of mechanical resonators," Phys. Rev. B 69. 125339/1-12 (2004).

[10] A.D. Armour, M.P. Blencowe and K.C. Schwab, "Entanglement and decoherence of a micromechanical resonator via coupling to a Cooperpair box,” Phys. Rev. Lett. 88, 148301/1-4. 